\title{
The Effectiveness of Single-Dose Fosfomycin as Antimicrobial Prophylaxis for Patients Undergoing Transrectal Ultrasound-Guided Biopsy of the Prostate
}

\author{
Şakir Ongün ${ }^{\mathrm{a}}$ Güven Aslan ${ }^{\mathrm{a}}$ Vildan Avkan-Oguz ${ }^{\mathrm{b}}$ \\ Departments of a Urology and ${ }^{b}$ Infectious Diseases and Clinical Microbiology, Dokuz Eylul University School of \\ Medicine, Izmir, Turkey
}

\section{Key Words}

Fluoroquinolone $\cdot$ Fosfomycin • Prophylaxis •

Prostate biopsy - Transrectal ultrasonography

Urinary tract infection

\begin{abstract}
Objectives: It was the aim of this study to evaluate the efficacy of single-dose fosfomycin prophylaxis as an alternative to fluoroquinolone-based prophylaxis in transrectal ultrasound-guided biopsy of the prostate (TRUSBP). Methods: We evaluated the records of 620 patients who had undergone TRUSBP from January 2010 to July 2011. Patients received a single dose of $3 \mathrm{~g}$ oral fosfomycin or a single dose of $500 \mathrm{mg}$ oral levofloxacin or $500 \mathrm{mg}$ oral ciprofloxacin twice daily administered for 5 days starting 1 day before the prophylaxis procedure. We reviewed all febrile and afebrile urinary tract infections (UTIS) within 1 month after TRUSBP. Results: Of the 620 patients, 19 (3.0\%) developed febrile UTI and 51 (8.2\%) developed afebrile UTI after biopsy. Of the 19 patients with febrile UTI, 1/19 (5.2\%) received fosfomycin, $4 / 19(21 \%)$ received levofloxacin and $14 / 19(73.6 \%)$ received ciprofloxacin for prophylaxis. Of the 51 patients with afebrile UTI, 4/51 (7.8\%) received fosfomycin, 8/51 (15.6\%) received levofloxacin and 39/51 (76.4\%) received ciprofloxacin for prophylaxis. There were a total of 10 fluoroquinolone-resis-
\end{abstract}

tant infections, and all of them occurred after the ciprofloxacin or levofloxacin prophylaxis and none after fosfomycin prophylaxis. Conclusions: The ease of use of fosfomycin, reducing the rate of fluoroquinolone-resistant infections and hospitalizations shows that it would be an alternative and effective drug for antimicrobial prophylaxis in TRUSBP.

Copyright $\odot 2012$ S. Karger AG, Basel

\section{Introduction}

Transrectal ultrasound-guided biopsy of the prostate (TRUSBP) is generally accepted as the standard procedure to detect prostate cancer. Although it is a safe procedure with low morbidity, it carries the risk of serious and potentially life-threatening infections.

Urinary tract infection (UTI) is considered the second most frequently noted complication of prostate biopsy, after bleeding complications. It has been described as a minor or major complication, depending on its severity. Although noncomplicated or afebrile UTIs frequently occur after biopsy (1.2-11.3\%), complicated or febrile UTIs are also not uncommon (1.4-4.5\%) $[1,2]$. When febrile UTI does occur, it is typically serious and leads to hospitalization.

\section{KARGER \\ Fax +4161306 1234 \\ E-Mail karger@karger.ch}

www.karger.com (c) 2012 S. Karger AG, Basel

0042-1138/12/0894-0439\$38.00/0

Accessible online at:

www.karger.com/uin
Dr. Sakir Ongün

Dokuz Eylul University School of Medicine

Department of Urology

Izmir (Turkey)

E-Mail sakirongun@hotmail.com 
The hospital admission rates for complications following TRUSBP have increased dramatically during the last 10 years primarily due to an increasing rate of infection-related complications. The hospital admission rate for infection-related reasons within 30 days of the procedure increased from $1.0 \%$ in 1996 to $4.1 \%$ in 2005 [3]. To reduce these infections, antimicrobial prophylaxis is generally considered the standard of care because it has been shown to decrease the rates of infection-related complications $[4,5]$ and is recommended by the European Association of Urology guidelines and a recently updated American Urological Association best practice policy statement $[6,7]$. The majority of urologists in Europe prescribe fluoroquinolone prophylaxis in TRUSBP [8]. Because fluoroquinolones have a broad antimicrobial spectrum, the use or overuse of these drugs by physicians has led to increasing fluoroquinolone-resistant pathogens. Multiple reports describe an emergent trend of increasing bacterial resistance and infection-related complications after prostate biopsies $[3,9,10]$. When patients present with post-prostate biopsy infective symptoms, almost $50 \%$ are associated with fluoroquinoloneresistant pathogens. The increasing number of fluoroquinolone-resistant pathogens points to the following question: do we need an alternative to fluoroquinolones for prophylaxis in TRUSBP? Escherichia coli is the most common organism implicated in post-biopsy infection, and $90 \%$ of Enterobacteriaceae isolates with advanced resistance to antimicrobial drugs are susceptible to fosfomycin [11]. Only few articles in the literature reported the use of fosfomycin for prophylaxis in urologic surgeries, with a prevention rate of $90.8 \%$ [12]. To our knowledge, use of fosfomycin for prophylaxis in prostate biopsy has not been addressed in clinical trials. The aim of this study was to evaluate the efficacy of a single-dose fosfomycin against a single dose of levofloxacin for prophylaxis in TRUSBP. The 5-day regime of oral ciprofloxacin prophylaxis is used as control group.

\section{Materials and Methods}

A retrospective evaluation was performed of the records of all patients who underwent TRUSBP at our institution from 1 January 2010 to 31 July 2011. Between 1 January 2010 and 31 December 2010, the patients who underwent TRUSBP received $500 \mathrm{mg}$ oral ciprofloxacin twice daily for 5 days, starting 1 day before the procedure. Routinely, a dipstick urinalysis (leukocyte esterase test and nitrite test), microscopic urinalysis and urine culture were performed on each patient before TRUSBP. Patients with either positive result did not undergo TRUSBP (the biopsy was rescheduled after appropriate culture-based treatment, if bacteriuria was identified). After reviewing the bacteriology of our post-TRUSBP UTIs within that period, our fluoroquinolone-resistant $E$. coli (FREc) rates were elevated. We changed our antibiotic prophylaxis protocol starting 1 January 2011. Between 1 January 2011 and 31 July 2011, consecutive patients received either a single dose of oral $500 \mathrm{mg}$ levofloxacin $60 \mathrm{~min}$ before the procedure or a single dose of fosfomycin (orally, $3 \mathrm{~g}$ ) the night before the procedure. Anticoagulant agents or acetylsalicylic acid were stopped 7 days before TRUSBP.

Biopsy indications were an elevated prostate-specific antigen (PSA) level $(>2.5 \mathrm{ng} / \mathrm{ml})$ and/or abnormal digital rectal examination and abnormal findings in the first prostate biopsy pathology that necessitated a repeat biopsy such as the presence of atypical glands. The latter was performed at least 6 weeks apart. All patients received a self-administered fleet enema (sodium phosphate and dibasic sodium phosphate) the night before the biopsy, with $2 \%$ lidocaine jelly instilled intrarectally for local anesthesia. With the patient in the left decubitus position, transrectal ultrasound was performed by the urologist with a multi-planar multifrequency probe $(75 \mathrm{MHz})$ attached to the ultrasound scanner. Prostate biopsies (10-12 cores) were taken using an automated biopsy gun with a disposable 18 gauge $\times 30 \mathrm{~cm}$ biopsy needle. Prostate volume was calculated using the prostate ellipsoid formula: volume $(\mathrm{V})=0.52(\mathrm{~L} \times \mathrm{W} \times \mathrm{H})$ where $\mathrm{L}$ is the cephalocaudal diameter, $\mathrm{W}$ is the width and $\mathrm{H}$ is the antero-posterior diameter.

Ten cores were taken in the first and 12 cores in the rebiopsies routinely. All of the biopsies were performed by the same two urologists. Saturation biopsy (24 cores) and having an indwelling urethral catheter are known risk factors for UTI after TRUSBP, so these patients were excluded [13]. Having undergone urologic surgery in the last month was another exclusion criterion. After biopsy, all patients were informed about the possible complications. Patients were instructed to return to our hospital if they developed a fever of $>38.0^{\circ} \mathrm{C}$, chills or rigors, severe irritative voiding symptoms and macroscopic hematuria. All patients were informed to come to the visits at day 3 , as well as 1 and 4 weeks after the biopsy.

We scheduled visits within 1 month after TRUSBP as a cut-off to capture only infections that may have been related to prostate biopsy. Any events $>1$ month after prostate biopsy were considered unlikely to have been related to TRUSBP. Physical examination, urine test and urine culture were performed in all patients at the first and fourth week after biopsy. Afebrile UTI was defined as a fever $<38^{\circ} \mathrm{C}$ and dysuria accompanied by pyuria [14]. Pyuria was defined as the presence of $>10$ white blood cells in $1 \mathrm{~mm}^{3}$ of mid-stream urine. Febrile UTI was defined as a fever $>38^{\circ} \mathrm{C}$ accompanied by one symptom of the lower urinary tract (i.e., urgency, frequency, dysuria, or suprapubic tenderness), with or without a positive urine culture [14-16]. All patients with febrile UTI were hospitalized.

When the patients were hospitalized, non-urological causes of the symptoms were excluded. Physical examination included body temperature measurement, signs of epididymo-orchitis and abdominal examination. Other systems examined included the respiratory and cardiac systems. Laboratory tests included complete blood count, blood urea nitrogen, creatinine and urine and blood cultures (aerobic and anaerobic).

The cultures were repeated if patients remained febrile or unwell with elevated total white counts. Chest X-rays were also performed. The patients were started on empirical intravenous ceftri- 
Table 1. Patient population for both groups

\begin{tabular}{|c|c|c|c|c|c|c|}
\hline & \multirow{2}{*}{$\begin{array}{l}\text { Ciprofloxacin } \\
(\mathrm{n}=406)\end{array}$} & \multirow{2}{*}{$\begin{array}{l}\text { Levofloxacin } \\
(\mathrm{n}=110)\end{array}$} & \multirow{2}{*}{$\begin{array}{l}\text { Fosfomycin } \\
(\mathrm{n}=104)\end{array}$} & \multicolumn{3}{|c|}{$\mathrm{p}$ value } \\
\hline & & & & F vs. L & F vs. C & F vs. $\mathrm{C}+\mathrm{L}$ \\
\hline Age, years & $63.88 \pm 7.27[42-86]$ & $63.23 \pm 7.78[41-87]$ & $61.5 \pm 6.63[41-77]$ & 0.092 & 0.03 & 0.06 \\
\hline Prostate volume, $\mathrm{cm}^{3}$ & $48.98 \pm 24.18[10-170]$ & $46.70 \pm 25.30[15-150]$ & $46.09 \pm 22.57[16-128]$ & 0.852 & 0.271 & 0.354 \\
\hline Number of biopsy cores & $10.18 \pm 0.576[10-12]$ & $10.34 \pm 0.743[10-12]$ & $10.32 \pm 0.775[10-12]$ & 0.864 & 0.032 & 0.091 \\
\hline Repeat biopsy & $37(9.1)$ & $19(17.2)$ & $17(16.3)$ & 0.834 & 0.032 & 0.115 \\
\hline Diabetes mellitus & $46(11.3)$ & $7(6.3)$ & $16(15.3)$ & 0.033 & 0.2 & 0.1 \\
\hline Previous urologic surgery & $21(5.1)$ & $6(5.4)$ & $5(4.8)$ & 0.831 & 0.96 & 0.922 \\
\hline
\end{tabular}

Data are presented as the mean \pm standard deviation with ranges in brackets, or as number of cases with percentages in parentheses. F = Fosfomycin; $\mathrm{L}=$ levofloxacin; $\mathrm{C}$ = ciprofloxacin.

Table 2. Microbiological characteristics and culture results of patients with afebrile UTI $(n=51 / 620,8.2 \%)$

\begin{tabular}{|c|c|c|c|c|c|c|}
\hline & \multirow[t]{2}{*}{ Ciprofloxacin } & \multirow[t]{2}{*}{ Levofloxacin } & \multirow[t]{2}{*}{ Fosfomycin } & \multicolumn{3}{|l|}{$\mathrm{p}$ value } \\
\hline & & & & F vs. L & F vs. C & F vs. $\mathrm{C}+\mathrm{L}$ \\
\hline Patients & $39 / 406(9.6 \%)$ & $8 / 110(7.2 \%)$ & $4 / 104(3.8 \%)$ & 0.428 & 0.091 & 0.079 \\
\hline Positive urine culture & 5 & 3 & 2 & 0.526 & 0.434 & 0.677 \\
\hline Negative urine culture & 34 & 5 & 2 & & & \\
\hline Fluoroquinolone-sensitive E. coli & 5 & - & 1 & & & \\
\hline Fluoroquinolone-resistant $E$. coli & - & 3 & - & & & \\
\hline ESBL E. coli ${ }^{1}$ & - & 1 & - & & & \\
\hline Enterococcus spp. & - & - & 1 & & & \\
\hline
\end{tabular}

$\mathrm{F}=$ Fosfomycin; $\mathrm{L}=$ levofloxacin; $\mathrm{C}=$ ciprofloxacin. ${ }^{1}$ Also fluoroquinolone-resistant.

axone, which was adjusted according to the culture results. When the fever had settled, the antibiotic was switched to an oral form and patients were discharged with 2 weeks of oral antibiotics.

\section{Statistical Analysis}

$\chi^{2}$ analysis and Fisher's exact test were used to compare variables between various groups in the study for categorical variables. $\mathrm{p}<0.05$ was considered significant.

\section{Results}

From 1 January 2010 to 31 July 2011, 620 patients underwent TRUSBP. Between 1 January 2010 to 31 December 2010, 406 patients received ciprofloxacin prophylaxis. Between 1 January 2011 to 31 July 2011, 110 patients received levofloxacin and 104 patients received fosfomycin prophylaxis. The mean age of patients was $63.38 \pm 7.30$ years (range 41-87), PSA was $8.42 \pm 7.73 \mathrm{ng} / \mathrm{ml}$ (range $1.09-387)$ and prostate volume was $48.08 \pm 24.11 \mathrm{~cm}^{3}$ (range 10-170). Patient characteristics for the ciprofloxa- cin, levofloxacin and fosfomycin groups are shown in table 1. They were similar in terms of mean ages, indications for biopsy, prostate volume and the number of biopsy cores taken. The number of patients having received previous urologic surgery was also similar in both groups. Diabetic and repeat biopsy patient numbers in the fosfomycin group were significantly higher than in the fluoroquinolone groups $(\mathrm{p}<0.05)$.

Of the 620 patients, 19 (3.06\%) developed febrile UTI and 51 (8.2\%) afebrile UTI after prostate biopsy. We detected 11 positive urine cultures in the febrile UTI group and 10 in the afebrile UTI group. Numbers of febrile UTIs, afebrile UTIs and types of bacteria for each group are given in tables 2 and 3 . Of the 19 patients who developed febrile UTI, 1/19 (5.2\%) received fosfomycin, 4/19 (21\%) levofloxacin and 14/19 (73.6\%) ciprofloxacin for prophylaxis. Of the 51 patients with afebrile UTI, 4/51 (7.8\%) received fosfomycin, 8/51 (15.6\%) levofloxacin and 39/51 (76.4\%) ciprofloxacin for prophylaxis. No statistical differences were found between groups. 
Table 3. Microbiological characteristics and culture results of patients with febrile UTI ( $\mathrm{n}=19 / 620,3.0 \%)$

\begin{tabular}{|c|c|c|c|c|c|c|}
\hline & \multirow[t]{2}{*}{ Ciprofloxacin } & \multirow[t]{2}{*}{ Levofloxacin } & \multirow[t]{2}{*}{ Fosfomycin } & \multicolumn{3}{|l|}{$\mathrm{p}$ value } \\
\hline & & & & F vs. L & F vs. C & F vs. $\mathrm{C}+\mathrm{L}$ \\
\hline Patients & $14 / 406(3.4 \%)$ & $4 / 110(3.6 \%)$ & $1 / 104(0.9 \%)$ & 0.202 & 0.154 & 0.775 \\
\hline Positive urine culture & 9 & 1 & 1 & 0.736 & 0.361 & 0.700 \\
\hline Negative urine culture & 5 & 3 & - & & & \\
\hline Fluoroquinolone-sensitive E. coli & 2 & 1 & 1 & & & \\
\hline Fluoroquinolone-resistant E. coli & 7 & - & - & & & \\
\hline ESBL E. coli ${ }^{1}$ & 6 & - & - & & & \\
\hline Enterococcus spp. & - & - & - & & & \\
\hline Positive blood culture ${ }^{2}$ & 3 & - & - & & & \\
\hline
\end{tabular}

$\mathrm{F}=$ Fosfomycin; $\mathrm{L}=$ levofloxacin; $\mathrm{C}=$ ciprofloxacin.

${ }^{1}$ All of these were also fluoroquinolone-resistant. ${ }^{2}$ All of these were ESBL E. coli as well as fluoroquinolone-resistant.

Of the 19 patients who developed febrile UTI, E. coli was detected in urine culture in 11 patients, $4(36.4 \%)$ of which were fluoroquinolone sensitive and 7 (63.6\%) with FREc. We detected extended-spectrum $\beta$-lactamase (ESBL) E. coli in 6 (85\%) patients with positive urine culture for FREc. None of the patients who received fosfomycin prophylaxis had positive urine culture for FREc. All of the patients with positive urine culture for FREc received ciprofloxacin prophylaxis. Three patients in the febrile UTI group had positive blood cultures, and ESBL E. coli was detected in all blood cultures. Of the 51 patients who developed afebrile UTI, E. coli was detected in urine culture in 9 patients, $3(33.3 \%)$ of which were fluoroquinolone sensitive and 6 (66.6\%) with FREc. None of the patients who received fosfomycin prophylaxis had positive urine culture for FREc. All of the patients with positive urine culture for FREc received levofloxacin prophylaxis. Out of 620 patients, 21 (3.3\%) developed documented UTI (coexistence of relative symptoms, leukocytosis, increased CRP and positive urine culture). Of the 21 patients who developed documented UTI, 3/21 (14.2\%) received fosfomycin, 4/21 (19\%) levofloxacin and 14/21 (66.6\%) ciprofloxacin prophylaxis. No statistical differences were found between the groups (the p value between levofloxacin and fosfomycin was 0.531 , between fosfomycin and ciprofloxacin 0.531 , and between levofloxacin and ciprofloxacin 0.557). The mean age of the 19 patients hospitalized for post-TRUSBP febrile UTI was $66 \pm 5.85$ years (range $54-76)$. The mean PSA level was $5.22 \pm 2.48 \mathrm{ng} / \mathrm{ml}$ (range 2.3-11) and the mean prostate volume was 51.1 \pm $19.57 \mathrm{~cm}^{3}$ (range 21-111). Three patients had diabetes mellitus and none of them had previous urologic surgery. The mean number of cores of prostate biopsies was 10.1, and only 1 patient had a repeat biopsy. The mean interval between biopsy and presentation to the emergency room with fever and urinary symptoms was $3.2 \pm 2.6$ days (range 1-10). Mean hospitalization time was $7.2 \pm 5.9$ days (range 2-25). One of 3 patients with positive blood culture remained in the hospital for 25 days and he stayed 3 weeks in the intensive care unit during this period. Six patients with positive urine culture with ESBL E. coli remained in the hospital for $13.3 \pm 5.8$ days (range 10-25) and they took $1 \mathrm{~g}$ ertapenem intravenously for 10 days.

When we detected fluoroquinolone-sensitive E. coli or negative cultures, we discharged patients with oral antibiotics for 2 weeks. The mean length of hospitalization was 2 days. For all patients, the 30 -day mortality rate was zero. After the introduction of fosfomycin, the number of hospitalization secondary to febrile UTI was reduced from 3.4 to $0.9 \%$. This represented a $>50 \%$ reduction, but the difference was not statistically significant. Febrile and afebrile UTIs were not detected with FREc or ESBL E. coli after the introduction of fosfomycin. However, the hospitalization rates secondary to febrile UTI between fosfomycin and fluoroquinolones were not statistically significant.

\section{Discussion}

TRUSBP is a common urological procedure performed in every center, but no standard antimicrobial prophylaxis regimen has yet been identified [17]. Fluoroquinolones are the most commonly used antibiotics recommended by guidelines for prophylaxis because they have been shown to decrease the rates of infection-related complications [4]. The efficacy of fluoroquinolones was initially hypothe- 
sized to result from their potent activity against a large spectrum of clinically relevant pathogens, especially E. coli. However, the common use of fluoroquinolones created another problem - fluoroquinolone resistance. Durham et al. [18] created a model to predict the future rate of fluoroquinolone-resistant E. coli in different countries. For the United States and Europe, $45 \%$ of E. coli can be expected to be fluoroquinolone-resistant in 2013. After prostate biopsy, the infection rate is $2-3 \%$, but positive culture for FREc in these patients is $55 \%$ [16]. In other studies, FREc is $100 \%$ positive in cultures and $55.6 \%$ of them are positive for extended-spectrum lactamases [19]. Recently, Lange et al. [20] reported an incidence of 96\% FREc with post-biopsy sepsis. Another study showed that in patients with infective complications and positive urine and/or blood cultures, $89 \%$ had E. coli, $90 \%$ of which were fluoroquinolone-resistant [9]. In this study, of the E. coli isolates from febrile UTI patients with positive culture results, $63 \%$ (7/11) were resistant to fluoroquinolones, and 54\% (6/11) were positive for ESBL. All the ESBL E. coli isolates were resistant to fluoroquinolones. Positive urine culture rates for FREc were similar to the results of previous studies [16]. Increasing resistance rates to fluoroquinolones in E. coli is a serious problem and fluoroquinolone-resistant infections after TRUSBP are increasing $[9,19,21]$. None of the patients receiving fosfomycin prophylaxis had a positive urine culture for FREc. All of the patients with positive urine culture for FREc had received ciprofloxacin for their prophylaxis protocol.

The high frequency of multiple antibiotic resistances among ESBL strains limits the therapeutic alternatives and the possibility of administering adequate empirical therapy. Patients with bacteremia caused by ESBL strains may have greater mortality. Inadequate empirical antimicrobial therapy is an independent risk factor for mortality in these patients [22]. In our study of patients with positive blood cultures, ESBL E. coli strains were isolated (3/3) and all were receiving ciprofloxacin prophylaxis; their 30 -day mortality rate was zero. All of the patients who received levofloxacin and fosfomycin prophylaxis had negative blood cultures. The overall hospitalization rate in our series was $3.0 \%$, similar to rates already reported in the literature $[3,9,23]$. The overall incidence of febrile UTIs in our study was $3.0 \%(19 / 620)$. This result was similar to previously reported rates $[8,18,19]$. Febrile UTI due to the fluoroquinolone prophylaxis was 3.4\% (18/516) and 0.9\% $(1 / 104)$ due to fosfomycin. The reduction was $>50 \%$ but it was not statistically significant, mostly because our sample size for the fosfomycin group was small. The febrile UTI rates between levofloxacin and ciprofloxacin pro- phylaxis was similar. Interestingly, there was no FREc in the levofloxacin group, but in the ciprofloxacin group, 7/14 patients showed FREc. We believe that this difference occurred because levofloxacin penetrates better to the prostate than ciprofloxacin, as recently reported by $\mathrm{Bu}-$ litta et al. [24]. The choice of fosfomycin as the agent to be included in the study was based on numerous published data reporting that $E$. coli is the most common organism implicated in post-biopsy infection and that the $90 \%$ of Enterobacteriaceae isolates with advanced resistance to antimicrobial drugs are susceptible to fosfomycin [11]. Urinary concentrations may exceed $2,000 \mathrm{mg} / \mathrm{l}$ after administration of a single oral dose of $3 \mathrm{~g}$ fosfomycin [25]. Peak urinary concentrations occur within $4 \mathrm{~h}$ of dosing, and urinary levels of fosfomycin remain high for a prolonged period (over $24 \mathrm{~h}$ ); thus, these levels are sufficient to inhibit most urinary pathogens [25]. We performed all the TRUSBG in the morning and finished by 12.00 p.m., so the patients received fosfomycin prophylaxis the night before the procedure $[25,26]$. According to our knowledge, this article is the first in the literature on the use of fosfomycin for prophylaxis in TRUSBP. Only a few articles on the use of fosfomycin for prophylaxis in urologic surgeries have a $90.8 \%$ prevention rate [12]. Compared with fluoroquinolones, imipenem and cephalosporins, we have demonstrated much lower resistance rates, with no imipenem resistance. But the inclusion of such agents in a routine prebiopsy preparation protocol will involve greater expenses and difficulties of injections. However, fosfomycin is easily orally administered the day before biopsy.

Our study has several important limitations. First, it is a retrospective study using the database and medical records of the patients enrolled. Second, the size of the groups with infection was limited because of the low incidence of complications in general. The use of fluoroquinolones in the last year increased the risk of FREc complications $[19,27]$. In our clinic, we were not questioning patients about the past use of fluoroquinolones before the biopsy which could be the third limitation of our study.

Despite all these limitations, antimicrobial prophylaxis using fosfomycin is more effective than fluoroquinolone-based prophylaxis for patients undergoing TRUSBP. Ease of use of fosfomycin, low infection rates, reducing the rates of fluoroquinolone-resistant infections and hospitalization shows that it would be an alternative and effective drug for antimicrobial prophylaxis in TRUSBP. The need for prospective randomized trials with an increased number of patients is inevitable. 


\section{Acknowledgements}

We acknowledge all the staff of the Dokuz Eylul University School of Medicine Urology Department.

\section{Disclosure Statement}

The authors declare no conflict of interest and no funding has been received for this work.

\section{References}

1 Djavan B, Waldert M, Zlotta A, Dobronski P, Seitz C, Remzi M, et al: Safety and morbidity of first and repeat transrectal ultrasoundguided prostate needle biopsies: results of a prospective European prostate cancer detection study. J Urol 2001;166:856-860.

$>2$ Aus G, Ahlgren G, Bergdahl S, Hugosson J: Infection after transrectal core biopsies of the prostate - risk factors and antibiotic prophylaxis. Br J Urol 1996;77:851-855.

3 Nam RK, Saskin R, Lee Y, Liu Y, Law C, Klotz $\mathrm{LH}$, et al: Increasing hospital admission rates for urological complications after transrectal ultrasound guided prostate biopsy. J Urol 2010;183:963-969.

4 Kapoor DA, Klimberg IW, Malek GH, Wegenke JD, Cox CE, Patterson AL, et al: Single-dose ciprofloxacin versus placebo for prophylaxis during transrectal prostate biopsy. Urology 1998;52:552-558.

$\checkmark 5$ Aron M, Rajeev TP, Gupta NP: Antibiotic prophylaxis for transrectal needle biopsy of the prostate: a randomized controlled study. BJU Int 2000;85:682-685.

6 European Association of Urology: Prostate cancer; in European Association of Urology: The European Association of Urology Guidelines. Arnhem, 2010.

7 Best Practice Policy Statement on Urologic Surgery Antimicrobial Prophylaxis. http:// www.auanet.org/content/media/antimicroprop08.pdf (accessed May 12, 2012).

$\checkmark 8$ Fink KG, Schmid HP, Paras L, Schmeller NT: Prostate biopsy in Central Europe: results of a survey of indication, patient preparation and biopsy technique. Urol Int 2007;79:6066.

$>9$ Feliciano J, Teper E, Ferrandino M, Macchia RJ, Blank W, Grunberger I, et al: The incidence of fluoroquinolone resistant infections after prostate biopsy - are fluoroquinolones still effective prophylaxis? J Urol 2008; 179:952-955.

10 Shigehara K, Miyagi T, Nakashima T, Shimamura M: Acute bacterial prostatitis after transrectal prostate needle biopsy: clinical analysis. J Infect Chemother 2008;14:40-43.
11 Falagas ME, Kastoris AC, Kapaskelis AM, Karageorgopoulos DE: Fosfomycin for the treatment of multidrug-resistant, including extended-spectrum $\beta$-lactamase-producing, Enterobacteriaceae infections: a systematic review. Lancet Infect Dis 2010;10:43-50.

12 Ishizaka K, Kobayashi S, Machida T, Yoshida $\mathrm{K}$ : Randomized prospective comparison of fosfomycin and cefotiam for prevention of postoperative infection following urological surgery. J Infect Chemother 2007;13:324331.

13 Simsir A, Kismali E, Mammadov R, Gunaydin G, Cal C: Is it possible to predict sepsis, the most serious complication in prostate biopsy? Urol Int 2010;84:395-399.

14 Whittam BM, Thomasch JR, Makari JH, Tanaka ST, Thomas JC, Pope JC 4th, et al: Febrile urinary tract infection after ureteroneocystostomy: a contemporary assessment at a single institution. J Urol 2010;183:688692.

15 Horan TC, Gaynes RP: Surveillance of nosocomial infections; in Mayhall CG (ed): Hospital Epidemiology and Infection Control, ed 3. Philadelphia, Lippincott, Williams and Wilkins, 2004, pp 1659-1702.

-16 Zaytoun OM, Vargo EH, Rajan R, Berglund R, Gordon S, Jones JS: Emergence of fluoroquinolone-resistant Escherichia coli as cause of postprostate biopsy infection: implications for prophylaxis and treatment. Urology 2011;77:1035-1042.

17 Shandera KC, Thibault GP, Deshon GE Jr: Variability in patient preparation for prostate biopsy among American urologists. Urology 1998;52:644-646.

18 Durham LK, Ge M, Cuccia AJ, Quinn JP: Quinn modeling antibiotic resistance to project future rates: quinolone resistance in Escherichia coli. Eur J Clin Microbiol Infect Dis 2010;29:353-356.

19 Akduman B, Akduman D, Tokgöz H, Erol B, Türker T, Ayoğlu F, et al: Long-term fluoroquinolone use before the prostate biopsy may increase the risk of sepsis caused by resistant microorganisms. Urology 2011;78:250-256.
20 Lange D, Zappavigna C, Hamidizadeh R, Goldenberg SL, Paterson RF, Chew BH: Bacterial sepsis after prostate biopsy - a new perspective. Urology 2009;74:1200-1205.

21 Ozden E, Bostanci Y, Yakupoglu KY, Akdeniz E, Yilmaz AF, Tulek N, et al: Incidence of acute prostatitis caused by extended-spectrum beta-lactamase-producing Escherichia coli after transrectal prostate biopsy. Urology 2009;74:119-123.

22 Hyle EP, Lipworth AD, Zaoutis TE, Nachamkin I, Bilker WB, Lautenbach E: Impact of inadequate initial antimicrobial therapy on mortality in infections due to extendedspectrum beta-lactamase-producing Enterobacteriaceae: variability by site of infection. Arch Intern Med 2005;165:1375-1380.

23 Raajimakers R, Kirkels WJ, Roobol MJ, Wildhagen MF, Schrder FH: Complication rates and risks factors of 5802 transrectal ultrasound-guided sextant biopsies of the prostate within a population-based screening program. Urology 2002;60:826-830.

24 Bulitta JB, Kinzig M, Naber CK, Wagenlehner FM, Sauber C, Landersdorfer CB, et al: Population pharmacokinetics and penetration into prostatic, seminal, and vaginal fluid for ciprofloxacin, levofloxacin, and their combination. Chemotherapy 2011;57:402416.

25 Raz R: Fosfomycin: an old-new antibiotic. Clin Microbiol Infect 2012;18:4-7.

26 Selvaggi FP, Battaglia M, Grossi FS, Disabato G, Cormio L: Oral prophylaxis with fosfomycin trometamol in transurethral prostatectomy and urological maneuvers: literature review and personal experience. Infection 1992;20(suppl 4):321-324.

27 Arslan H, Azap OK, Ergönül O, Timurkaynak F: Urinary Tract Infection Study Group. Risk factors for ciprofloxacin resistance among Escherichia coli strains isolated from community-acquired urinary tract infections in Turkey. J Antimicrob Chemother 2005;56:914-918. 